\title{
Diphenhydramine induces melanoma cell apoptosis by suppressing STAT3/MCL-1 survival signaling and retards B16-F10 melanoma growth in vivo
}

\author{
CHI-HUNG R. OR ${ }^{2,3}$, HONG-LIN SU ${ }^{2,4}$, WEE-CHYAN LEE ${ }^{1}$, SHU-YI YANG ${ }^{1}$, \\ CHEESANG $\mathrm{HO}^{3}$ and CHIA-CHE CHANG ${ }^{1,2,4,5-8}$
}

\begin{abstract}
${ }^{1}$ Institute of Biomedical Sciences and ${ }^{2}$ Department of Life Science, National Chung Hsing University, Taichung 40227; ${ }^{3}$ Department of Anesthesiology, Kuang Tien General Hospital, Dajia Branch, Taichung 43761; ${ }^{4} \mathrm{Ph} . D$. Program in Translational Medicine, ${ }^{5}$ Agricultural Biotechnology Center and ${ }^{6}$ Rong Hsing Research Center for Translational Medicine, National Chung Hsing University, Taichung 40227; ${ }^{7}$ Department of Biotechnology, Asia University, Taichung 41354;

${ }^{8}$ Department of Medical Research, China Medical University Hospital, Taichung 40447, Taiwan, R.O.C.
\end{abstract}

Received May 12, 2016; Accepted June 8, 2016

DOI: $10.3892 /$ or.2016.5201

\begin{abstract}
Melanoma is the most aggressive skin malignancy with a high rate of mortality and is frequently refractory to many therapeutics, thus demanding the discovery of novel effective anti-melanoma agents. Diphenhydramine (DPH) is an $\mathrm{H} 1$ histamine receptor antagonist and a relatively safe drug. Previous studies have revealed the in vitro cytotoxicity of DPH against melanoma cells, but the mechanisms involved concerning its cytotoxicity and the in vivo anti-melanoma effect remain unknown. We herein present the first evidence supporting that DPH is selectively proapoptotic for a panel of melanoma cell lines irrespective of $\mathrm{BRAF}^{\mathrm{V} 600 \mathrm{E}}$ status while sparing normal melanocytes. Of note, DPH effectively suppressed tumor growth and prolonged the length of survival of mice bearing B16-F10 melanoma. Mechanistic investigation further revealed that DPH downregulated antiapoptotic MCL-1, whereas MCL-1 overexpression impeded the proapoptotic action of DPH. Moreover, DPH attenuated STAT3 activation, as evidenced by the reduced levels of tyrosine 705-phosphorylated STAT3. Notably, ectopic expression of constitutively active STAT3 mutant reduced DPH-induced apoptosis but also protected MCL-1 from downregulation by DPH, illustrating that DPH impairs STAT3 activation to block STAT3-mediated induction of MCL-1 in eliciting apoptosis. Collectively, we for the first time validate the in vivo anti-melanoma effect of DPH and also establish DPH as a drug targeting STAT3/MCL-1 survival signaling pathway to induce
\end{abstract}

Correspondence to: Professor Chia-Che Chang, Institute of Biomedical Sciences, National Chung Hsing University, 145 Xingda Road, Taichung 40227, Taiwan, R.O.C.

E-mail: chia_che@dragon.nchu.edu.tw; chiachechang@gmail.com

Key words: diphenhydramine, melanoma, STAT3, MCL-1, apoptosis, B16-F10 melanoma model apoptosis. Our discovery therefore suggests the potential to repurpose DPH as an anti-melanoma therapeutic agent.

\section{Introduction}

Melanoma is the most aggressive skin malignancy, accounting for more than $80 \%$ of skin cancer-related deaths (1). An increasing incidence of melanoma has been observed globally despite early detection, appropriate resection and adjuvant therapy (2). The prognosis of advanced melanoma is poor, with a median survival of 6-9 months. Currently, treatment options for advanced melanoma are limited. Mutations in $B R A F$ (mostly V600E) are commonly present in $40-60 \%$ of cutaneous melanoma (3) and are associated with brain and lung metastases (4), therefore representing a promising therapeutic target for melanoma. Patients harboring BRAF mutations indeed had shown clinical response to BRAF inhibitors such as vemurafenib. However, most of them had only a short-lived response with a progression-free survival of only 5 months, and resistance occurred eventually in most cases (5). Thus, development of novel effective chemotherapeutic agents used as a monotherapy or in combination with BRAF inhibitors is in urgent demand.

Diphenhydramine (DPH) is a first-generation antihistamine (H1 histamine receptor antagonist) developed in the late 1940s. It has been widely used for many different purposes in clinics due to its various pharmacological effects. Because of the relative safety of this drug, DPH was approved by the FDA for non-prescription use to treat allergies, insomnia, cough, motion sickness and itchiness (6). It can also be used to relieve extrapyramidal symptoms and treat chemotherapy-induced nausea/vomiting (7). Furthermore, as a sodium channel blocker, DPH can act as a local anesthetic $(8,9)$. In normal clinical dosage, the side effects of DPH are mild and well tolerated. The common side effects are drowsiness, dry mouth and increased heart rate.

Previous studies by Jangi et al have explored the anti-melanoma potential of DPH by showing its ability to induce in vitro 
cytotoxicity against four human melanoma cell lines (10). However, the mechanisms of action underlying DPH-induced cytotoxicity and the in vivo anti-melanoma effect of this drug remain unknown. In light of this, we herein provide the first evidence to demonstrate the selective proapoptotic action of DPH on melanoma cells while sparing normal melanocytes, establishing DPH as a drug targeting the STAT3/MCL-1 survival signaling pathway to induce apoptosis, and validate the in vivo anti-melanoma effect of DPH in a B16-F10 melanoma mouse model. Our discovery therefore suggests the potential to repurpose DPH as an anti-melanoma therapeutic agent.

\section{Materials and methods}

Chemicals. DPH was obtained from Sigma-Aldrich (St. Louis, MO, USA). The pan-caspase inhibitor z-VAD.fmk was purchased from Cayman Chemical (Ann Arbor, MI, USA).

Cell culture. Human melanoma cell lines A2058 (no. CRL-11147), A375.S2 (no. CRL-1872), MDA-MB-435S (no. HTB-129) and murine B16-F10 melanoma cell line [no. CRL-6475; all from American Type Culture Collection (ATCC), Manassas, VA, USA] were grown at $37^{\circ} \mathrm{C}$ and $5 \% \mathrm{CO}_{2}$ in the culture media recommended by ATTC. Primary human melanocytes were obtained from Invitrogen Life Technologies (Carlsbad, CA, USA) and grown in culture media according to the manufacturer's instructions. All culture media and supplements were purchased from Invitrogen Life Technologies.

Cell viability assay. DPH-elicited cytotoxicity was evaluated by the levels of cell viability after drug treatment using an MTS assay (Promega Corp., Madison, WI, USA), which was performed according to our established protocol (11). In the present study, cells were seeded onto 96 -well plates at a density of $8 \times 10^{3}$ cells/well, followed by drug treatment and subsequent MTS assay.

Annexin V/propidium iodide (PI) dual staining assay using flow cytometry. Apoptosis induced by DPH was determined by Annexin V/PI dual staining assay using an ApoTarget ${ }^{\text {TM }}$ Annexin V-FITC Apoptosis kit (BioSource International, Inc., Camarillo, CA, USA) following our reported protocol (11). Data acquisition and analysis were performed on FACScan flow cytometer (BD Biosciences, USA). Results are expressed as the mean \pm SEM of at least three independent experiments. Cells with Annexin V-positive staining are recognized as cells undergoing apoptosis.

Construction of the retroviral vector pBabe-based MCL-1 or STAT3-expressing plasmid for stable clone generation. The open reading frames (ORF) of human MCL-1 (Genbank accession no. NM_021960) were PCR-amplified from the first-strand cDNA pools of human colon adenocarcinoma cell line HCT116 using the following primer pair: MCL-1 forward, 5'-ACCggTTTTggCCTCAAAAgAAAC-3' and MCL-1 reverse, 5'-CAgTAAggCTATCTTATTAgATATg-3'. The ORF of the constitutive STAT3 mutant (A661C/N663C) was PCR-amplified using the plasmid pMXs-Stat3-C (plasmid \#13373; Addgene, Inc., Cambridge, MA, USA) as the template.
The PCR-amplified ORFs were then subcloned to the retroviral vector $\mathrm{pBabe}$.puro engineered to encode an in-frame $\mathrm{N}$-terminal hemagglutinin (HA) epitope (pBabe-HA). The resultant expression plasmids for MCL-1 (pBabe-HA-MCL-1) or STAT3 (pBabe-HA-STAT3-CA) were then subjected to production of retroviral particles for subsequent cell infection and puromycin selection for stable infectants according to our published protocol (11).

Immunoblot analysis. Immunoblotting was performed as previously described (11). Polyclonal antibodies against MCL-1, BCL-xL, BAX, HA epitope, total ERK, phospho-ERK (Thr202/Tyr204), total STAT3, phospho-STAT3 (Tyr705), and the cleaved forms of poly(ADP-ribose) polymerase (PARP), caspase-3, caspase- 8 and caspase-9 were all purchased from Cell Signaling Technology (Beverly, MA, USA). $\alpha$-tubulin antibody was purchased from GeneTex (Irvine, CA, USA). The signals were detected with an enhanced SuperSignal West Pico chemiluminescence kit (Pierce, Rockford, IL, USA).

In vivo anti-melanoma activity assay. The in vivo anti-melanoma effect of DPH was evaluated using the murine B16-F10 cell line-based preclinical melanoma model. Briefly, $3 \times 10^{5}$ B16-F10 cells were subcutaneously injected into the ventral flank of C57BL/6 mice to allow tumor growth for 8 days until reaching the size of $10 \mathrm{~mm}^{3}$. Tumor-bearing mice were then randomized into 2 groups $(n=6)$ and received an i.p injection of $100 \mu \mathrm{l}$ PBS or DPH $(20 \mathrm{mg} / \mathrm{kg})$. Mice were subjected to an i.p. injection every other day. Tumor volumes were calculated with a digital caliper as length $\mathrm{x}$ width $\mathrm{x}$ thickness $\mathrm{x} 0.5$ and expressed in $\mathrm{mm}^{3}$ before each treatment. Mice were euthanized when the tumor size reached $<2,000 \mathrm{~mm}^{3}$ in mean diameter. All treatments were performed according to the guidelines approved by IACUC of the National Chung Hsing University.

Statistical analysis. All data from in vitro experiments were expressed as means \pm standard error of the mean (SEM) from at least three independent experiments. Differences between groups were evaluated for statistical significance using a Student's t-test. A p-value $<0.05$ was regarded as the minimum criteria for statistical significance. As for the in vivo studies, the Kaplan-Meier method and log-rank test using GraphPad Prism software package version 5.0 (GraphPad Software, Inc., San Diego, CA, USA) were employed to compare the survival rates between vehicle controls and DPH-treated mice. Differences were considered significant at $\mathrm{p}<0.05$.

\section{Results}

DPH induces apoptosis-dependent cytotoxicity selectively in malignant melanoma cells while sparing normal melanocytes. To comprehensively explore the anti-melanoma activity of DPH, we started by testing the in vitro cytotoxic effect of DPH on a panel of melanoma cell lines, including murine B16-F10 cells (BRAF wild-type) and human A2058, A375. S2 and MDA-MB-435S cells (all carrying the BRAF ${ }^{\mathrm{V} 600 \mathrm{E}}$ mutation) in addition to normal human melanocytes. As shown in Fig. 1A, DPH was cytotoxic against all melanoma cell lines tested; in contrast, normal human melanocytes were refractory to DPH-elicited cell death. Additionally, 
A

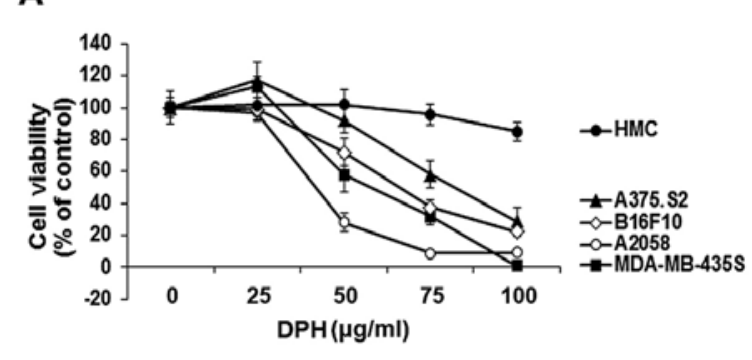

B

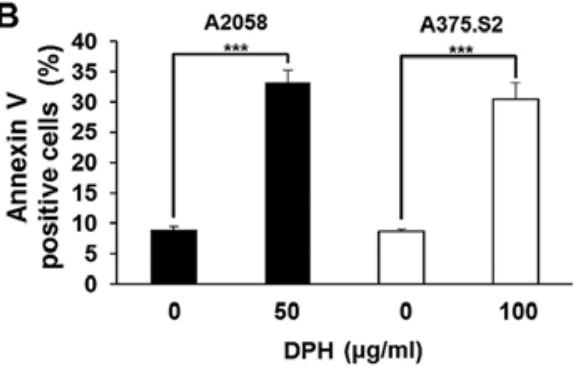

C

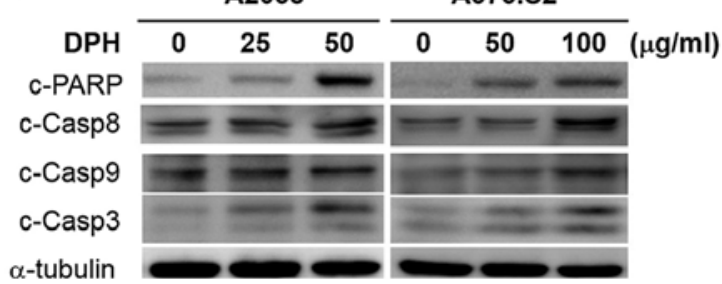

D
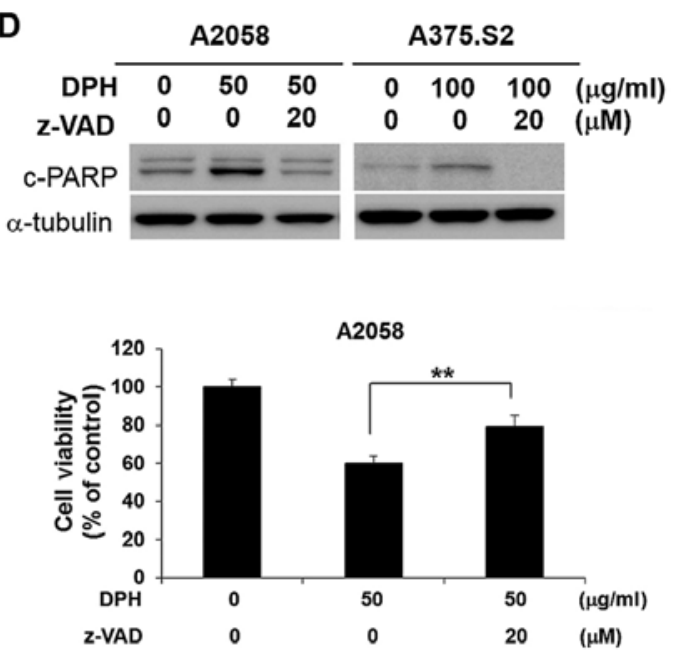

A375.S2

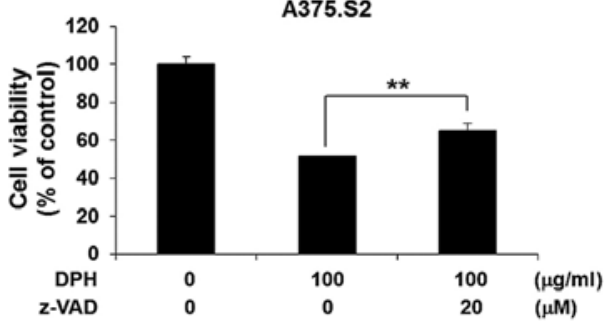

Figure 1. Diphenhydramine (DPH) induces apoptosis-dependent cytotoxicity selectively in melanoma cells. (A) Selective cytotoxic effect of DPH on melanoma cells with limited toxicity to normal melanocytes. Normal human melanocytes (HMC) (•), human melanoma cell lines A2058 (O), A375.S2 (ム) and MDA-MB-435S (ם), and murine melanoma cell line B16-F10 $(\diamond)$ were subjected to a 48-h treatment with DPH $(0-100 \mu \mathrm{g} / \mathrm{ml})$, followed by cell viability determination using an MTS assay. (B) Diphenhydramine enhances the Annexin V-positive (apoptotic) cell population. A2058 and A375.S2 cells were exposed to 50 and $100 \mu \mathrm{g} / \mathrm{ml}$ of diphenhydramine, respectively, followed by Annexin V/propidium iodide (PI) dual staining analyzed by flow cytometry. Shown here are the quantitative results of the levels of Annexin V-positive cell population after diphenhydramine stimulation. (C) DPH treatment leads to caspase activation. A2058 and A375.S2 cells treated with indicated concentrations of DPH were subjected to immunoblotting for the levels of cleaved forms of poly(ADP-ribose) polymerase (c-PARP), caspase-9 (c-Casp9), caspase-8 (c-Casp8) and caspase-3 (c-Casp3). $\alpha$-tubulin levels were used as the loading control. (D) Blockade of caspase activity attenuated DPH-induced cell death. A2058 and A375.S2 cells were pre-treated for $1 \mathrm{~h}$ with $20 \mu \mathrm{M}$ of $\mathrm{z}-\mathrm{VAD}$.fmk (Z-VAD), a pan-caspase inhibitor, followed by 24 -h treatment with DPH. Immunoblotting revealed a marked reduction in DPH-elicited PARP cleavage by z-VAD.fmk, confirming the blockade of caspase activity. $\alpha$-tubulin levels were used as the control for equal loading (upper panel). $z$-VAD.fmk pre-treatment significantly decreased DPH-induced cell death (middle and lower panels). ${ }^{* *} \mathrm{p}<0.01,{ }^{* * *} \mathrm{p}<0.001$.

we found that DPH treatment led to a marked increase in Annexin V-positive (apoptotic) cells in both the A2058 (from $8.96 \pm 0.49$ to $33.10 \pm 2.12 \%, \mathrm{p}<0.001$ ) and $\mathrm{A} 375 . \mathrm{S} 2$ (from $8.65 \pm 0.49$ to $30.36 \pm 2.68 \%, \mathrm{p}<0.001$ ) cell lines (Fig. 1B), illustrating the induction of apoptosis. Immunoblotting further revealed that DPH induced a dose-dependent increase in the levels of caspase-dependent cleavage of PARP in addition to the proteolytic processing and thus activation of caspase-9, caspase-8 and caspase-3 (Fig. 1C). These results thus confirmed the proapoptotic effect of DPH on melanoma cells. Noteworthy, pre-treatment with the pan-caspase inhibitor z-VAD.fmk significantly rescued A2058 and A375.S2 cells from DPH-induced cell death (Fig. 1D). Altogether, our results established the anti-melanoma effect of DPH through the induction of apoptosis-dependent cytotoxicity.

DPH suppresses the growth of B16-F10 melanoma in vivo. We next addressed whether the in vitro cytotoxicity of DPH accounts for its in vivo anti-melanoma effect. Using the well-established B16-F10 melanoma mouse model, we found that i.p. administration of DPH $(20 \mathrm{mg} / \mathrm{kg})$ evidently suppressed tumor growth compared to the vehicle control (Fig. 2A and B). Furthermore, the tumor volume was decreased to nearly $30 \%$ of the vehicle control 19 days after DPH administration (from $1750 \pm 176$ to $610 \pm 209 \mathrm{~mm}^{3}$, $\mathrm{p}<0.001)$. It is also noteworthy that DPH markedly prolonged the survival of the B16-F10-bearing mice (Fig. 2C). Overall, these results validated the in vivo anti-melanoma effect of $\mathrm{DPH}$.

MCL-1 downregulation is vital to DPH-induced melanoma cell apoptosis. The mechanism underlying the proapoptotic action of DPH was further investigated. We started by testing the effect of DPH on the expression of proteins responsible for the control of apoptosis initiation, including proapoptotic BAX and antiapoptotic BCL-xL and MCL-1. It is noteworthy that only MCL-1 was markedly reduced by DPH in both the A2058 and A375.S2 cells (Fig. 3A). Given that MCL-1 is fundamental for melanoma progression, relapse and drug resistance (12-14), the role of MCL-1 downregulation in DPH-elicited anti-melanoma activity was further examined in the A2058 and A375.S2 clones stably overexpressing MCL-1. 
A

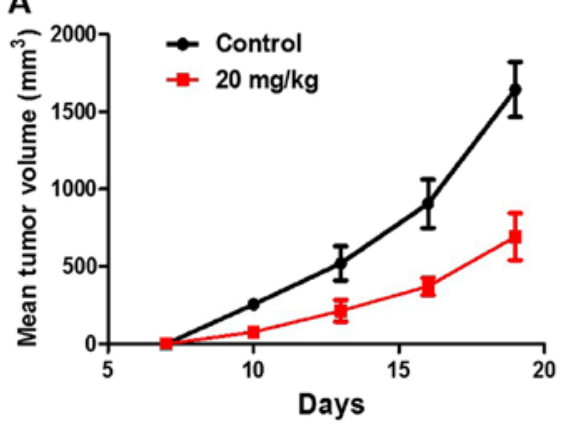

B

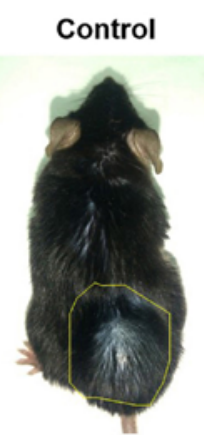

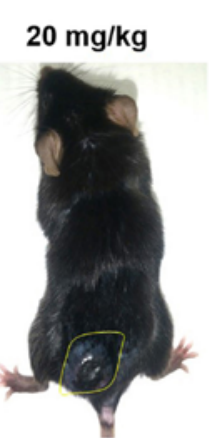

C

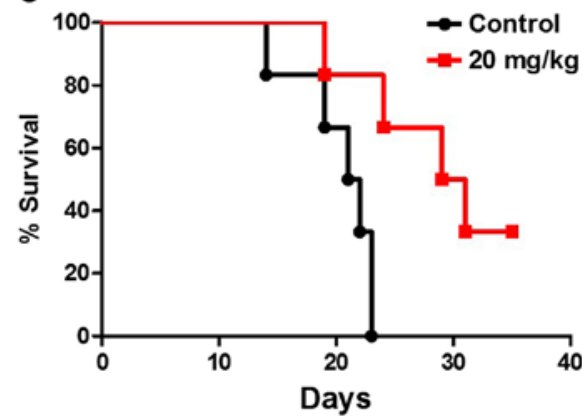

Figure 2. In vivo anti-melanoma effect of diphenhydramine (DPH). (A) DPH suppressed the growth of B16-F10 melanoma in C57BL/6 mice. B16-F10 melanoma-bearing mice received i.p. administration of DPH $(20 \mathrm{mg} / \mathrm{kg})$ every other day. The mean tumor volume was measured at the indicated number of days after tumor implant. (B) Representative tumors from the vehicle control and DPH-treated groups (day 21). Experiments were repeated twice with similar results. (C) DPH markedly prolonged the survival of B16-F10 melanoma-bearing mice. Shown here is the Kaplan-Meier survival curves of B16 melanomabearing mice for the vehicle control and DPH treatment groups $(n=6)$.

A
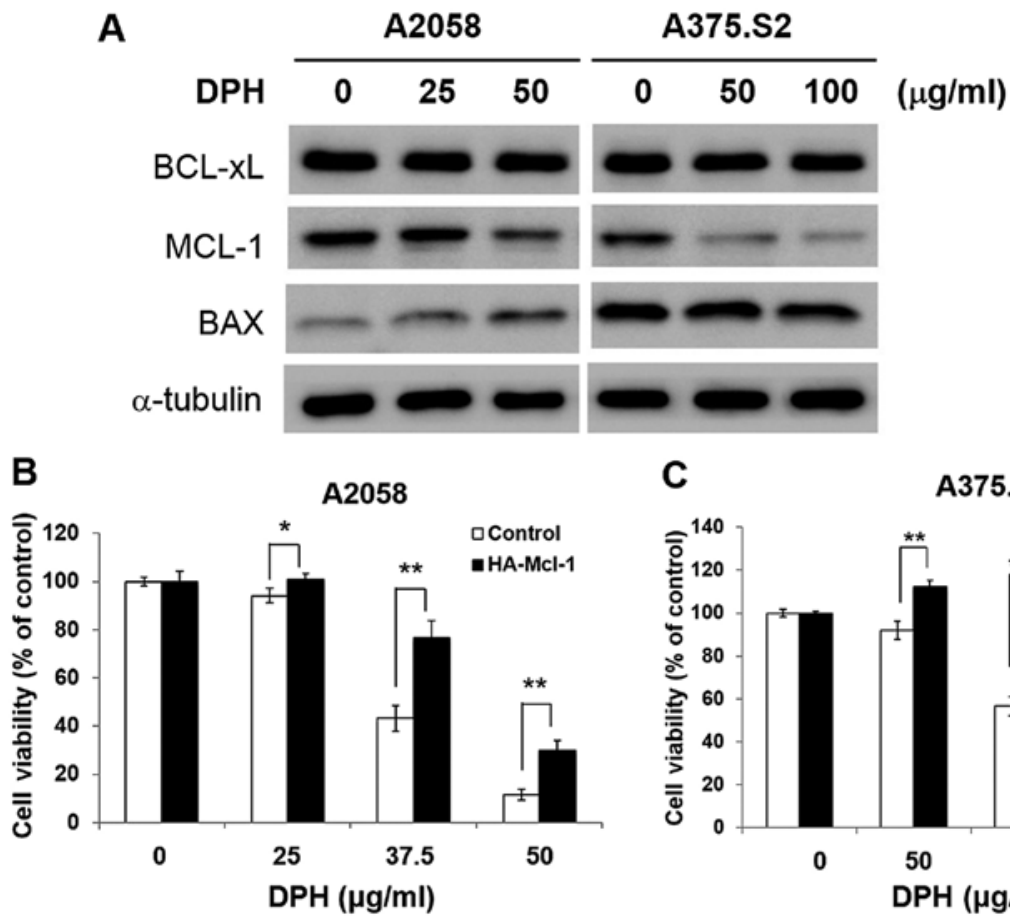

C A375.S2

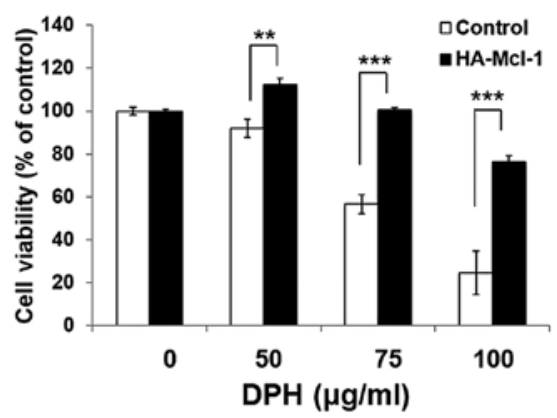

$\mathbf{D}$

A2058

E

A375.S2

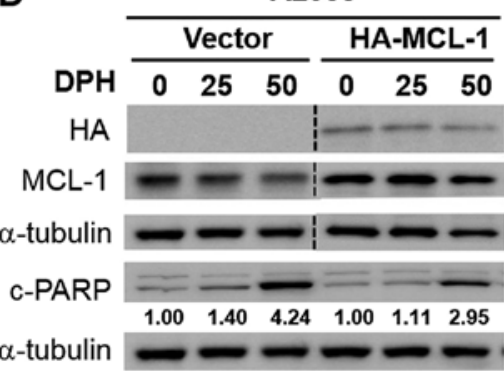

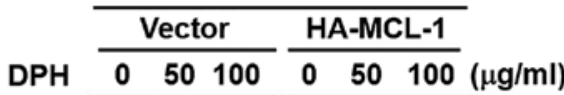

$\mathrm{HA}$

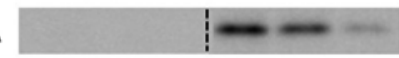

$\alpha$-tubulin $\longrightarrow-\infty=$

MCL-1

c-PARP

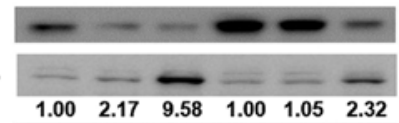

$\alpha$-tubulin

Figure 3. MCL-1 downregulation is essential for the efficient induction of melanoma cell apoptosis by diphenhydramine (DPH). (A) DPH downregulated MCL-1 in melanoma cells. A2058 and A375.S2 cells were treated with indicated doses of DPH, followed by immunoblotting to evaluate the levels of BCL-xL, MCL-1 and BAX. $\alpha$-tubulin levels served as the loading control. (B and C) Overexpression of MCL-1 effectively rescued cells from DPH-induced cell death. (B) A2058 and (C) A375.S2 clones stably overexpressing HA-tagged MCL-1 were treated with indicated concentrations of DPH for $48 \mathrm{~h}$, followed by the determination of cell viability. (D and E) Overexpression of MCL-1 supressed the proapoptotic action of DPH. Immunoblotting was used to evaluate the levels of the HA epitope and MCL-1 to confirm the overexpression of MCL-1 in respective stable clones of (D) A2058 and (E) A375.S2. Notably, the marked reduction in the levels of poly(ADP-ribose) polymerase (PARP) cleavage in MCL-1 stable clones highlighted the inhibition of DPH-elicited apoptosis by overexpression of MCL-1. The number of underlying c-PARP blots indicated the relative levels of signal densities compared to those of the drug-untreated control. $\alpha$-tubulin levels served as the control for equal loading. ${ }^{*} \mathrm{p}<0.05,{ }^{* *} \mathrm{p}<0.01,{ }^{* * *} \mathrm{p}<0.001$. 


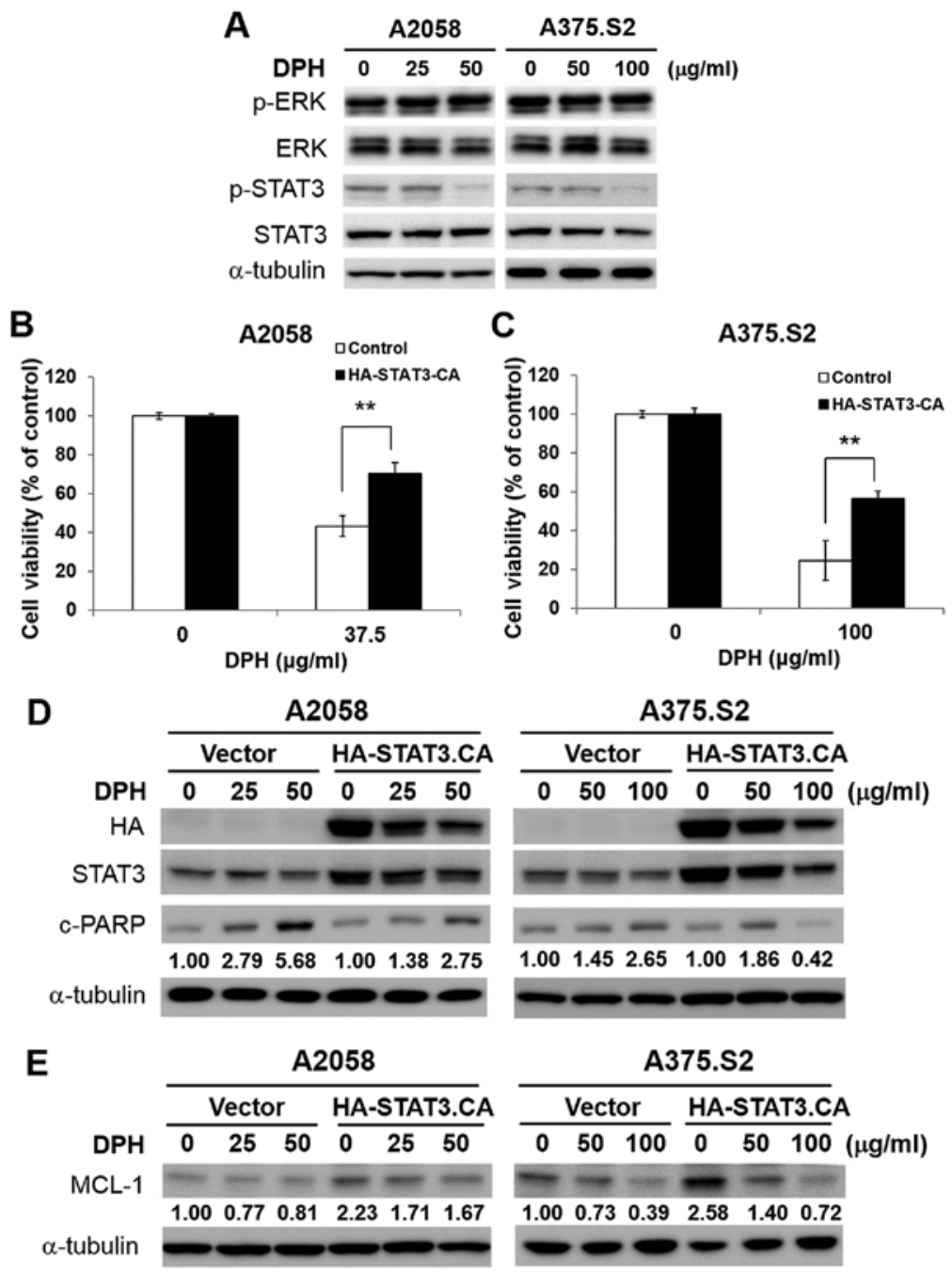

Figure 4. Diphenhydramine (DPH) impairs STAT3-mediated induction of MCL-1 to evoke melanoma cell apoptosis. (A) DPH attenuated STAT3 activation. A2058 and A375.S2 cells were subjected to a 24-h treatment with indicated doses of DPH, followed by immunoblotting to assess the levels of total ERK, phospho-ERK (Thr202/Tyr204), total STAT3 and Tyr705-phosphorylated STAT3. $\alpha$-tubulin levels were used as the loading control. (B and C) Constitutive STAT3 activation rescues cells from DPH-induced cell death. (B) A2058 and (C) A375.S2 stable clones overexpressing an HA epitope-tagged constitutively active STAT3 mutant (HA-STAT3-CA) were treated with 37.5 and $100 \mu \mathrm{g} / \mathrm{ml}$ of DPH, respectively, followed by cell viability determination. (D) Constitutive STAT3 activation impaired DPH-elicited apoptosis. Immunoblotting was used to determine the levels of the HA epitope and STAT3 to confirm HA-STAT3-CA overexpression in the A2058 and A375.S2 stable clones. The evident decrease in the levels of PARP cleavage in the HA-STAT3-CA stable clones illustrated the blockade of the proapoptotic action of DPH. The number of underlying c-PARP blots indicated the relative levels of signal densities compared to those of the drug-untreated control. $\alpha$-tubulin levels served as the control for equal loading. (E) Constitutive STAT3 activation protects MCL-1 from downregulation by DPH. Immunoblotting was performed to determine the levels of MCL-1 in the A2058 and A375.S2 HA-STAT3-CA stable clones with or without DPH treatment. The number of underlying MCL-1 blots indicated the relative levels of signal densities compared to those of the drug-untreated control. $\alpha$-tubulin levels served as the control for equal loading. ${ }^{* *} \mathrm{p}<0.01$.

We found that MCL-1 overexpression potently rescued cells from DPH-evoked cell death (Fig. 3B and C). Immunoblotting further revealed noticeable reduction in DPH-induced PARP cleavage in the MCL-1 stable clones (Fig. 3D and E), suggesting that MCL-1 overexpression blunts DPH-elicited apoptosis. Collectively, we conclude that downregulation of MCL-1 is integral to the proapoptotic action of DPH.

DPH impairs STAT3/MCL-1 survival signaling in melanoma cells. We next explored how DPH downregulates MCL-1. It has been shown that oncogenic $\mathrm{BRAF}^{\mathrm{V} 600 \mathrm{E}}$-initiated persistent ERK signaling upregulates MCL-1 (15). However, immunoblotting showed limited alteration in the levels of phospho-ERK (Thr202/Tyr204) following DPH treatment (Fig. 4A), suggesting that DPH failed to inhibit ERK signaling to decrease MCL-1. Alternatively, we tested the effect of DPH on the activation of STAT3 [revealed by phosphorylation of tyrosine 705 (p-Y705-STAT3)], considering that MCL-1 is a known transcriptional target of STAT3 (16). In addition, constitutive activation of STAT3 is associated with melanoma tumorigenesis, progression and chemoresistance through the upregulation of the transcription of genes promoting cell proliferation, survival, angiogenesis and metastasis $(12,17,18)$. Our results indicated that DPH impeded constitutive STAT3 activation, as evidenced by the decrease in the levels of p-Y705-STAT3 in both the A2058 and A375.S2 cells (Fig. 4A). Furthermore, overexpression of a constitutively active STAT3 mutant (HA-STAT3-CA) effectively protected cells from DPH-induced cell death (Fig. 4B and C), likely through the reduction of apoptosis (Fig. 4D), thus illustrating an essential 


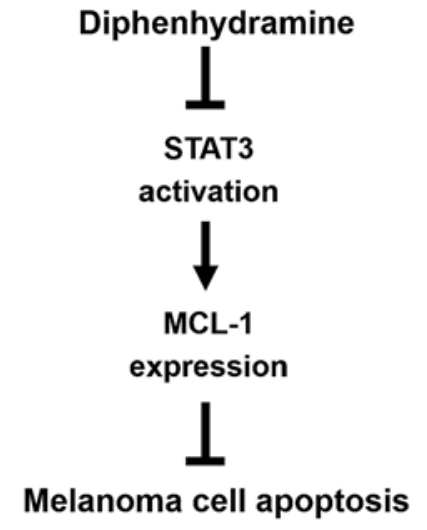

Figure 5. Schematic model of the STAT3/MCL-1 axis of survival in diphenhydramine (DHP)-elicited melanoma cell apoptosis. DHP attenuates STAT3 activation to impair STAT3-mediated induction of MCL-1 for apoptosis induction in melanoma cells.

role of STAT3 blockage in the proapoptotic action of DPH. Moreover, HA-STAT3-CA-expressing cells displayed an increase in the basal levels of MCL-1 but also markedly restored MCL-1 expression levels following DPH treatment (Fig. 4E), indicating that DPH downregulates MCL-1 by impairing STAT3-mediated induction of MCL-1. Overall, we conclude that DPH likely blocks the STAT3-MCL-1 survival signaling pathway to induce apoptosis in melanoma cells.

\section{Discussion}

In this study, we present clear evidence supporting the in vitro and in vivo anti-melanoma activities of DPH. Particularly, we revealed that DPH induced apoptosis-dependent cytotoxicity against a panel of melanoma cell lines while sparing normal melanocytes (Fig. 1). We also verified a marked suppression of in vivo B16-F10 melanoma growth after DPH administration (Fig. 2). Mechanistically, DPH thwarts STAT3 activation to downregulate MCL-1, leading to the induction of apoptosis in melanoma cells (Fig. 5). To the best of our knowledge, this is the first report validating the in vivo anti-melanoma activity of DPH in addition to the role of the STAT3/MCL-1 survival signaling pathway in DPH-induced melanoma cell apoptosis.

Data presented here for the first time confirm that DPH is selectively cytotoxic in malignant melanoma cell lines (Fig. 1), hence highlighting its advantage as an anticancer agent. The molecular basis underlying this selective cytotoxicity is currently unknown. Notably, recent studies have revealed higher expression levels of MCL-1 in melanoma cell lines and also in tissue samples from melanoma patients compared to their normal counterparts (19). It is thus reasonable to presume that melanoma cells are more sensitive to the decrease in MCL-1-mediated survival signals than normal melanocytes. Along this line, DPH-elicited MCL-1 downregulation likely accounts for its selective cytotoxicity against melanoma cells.

We found that the anti-melanoma activity of DPH is attributed to the induction of apoptosis (Fig. 1). DPH was previously shown to evoke apoptosis in T-cell acute lymphoblastic leukemia cell lines (20), suggesting that apoptosis induction is a general mode of action underlying the anticancer effect of DPH. It has also been shown that additional H1 histamine receptor antago- nists such as terfenadine and astemizol were proven to induce apoptosis in melanoma cells (10). Surprisingly, terfenadine provokes melanoma cell apoptosis through an $\mathrm{H} 1$ histamine receptor-independent mechanism (21); likewise, the anticancer effect of astemizol is not entirely ascribed to its antihistamine activity (22). Given that DPH is both an $\mathrm{H} 1$ antihistaminic and a sodium channel blocker, further investigation is warranted in order to ascertain whether DPH induces apoptosis through inhibition of the H1 receptor activity or blockage of sodium channels, whose promoting roles in cancer cell proliferation and metastasis have been recently uncovered (23).

Our mechanistic exploration identified MCL-1 downregulation as a pivotal mode of action underlying DPH-elicited melanoma cell apoptosis (Fig. 3). Notably, high MCL-1 expression is fundamental for the progression, relapse and chemoresistance of multiple types of human cancer, including melanoma, therefore making MCL-1 a promising therapeutic target (12-14). Indeed, recent studies have validated MCL-1 targeting as an effective strategy to eradicate cancer cells which are dependent on MCL-1 for survival or chemoresistance $(19,24)$, leading to intensive efforts toward the discovery of novel MCL-1-targeted therapeutic agents. Our finding that DPH decreases MCL-1 expression therefore adds DPH to the growing list of novel MCL-1-targeted small molecules with great translation potential as anticancer agents.

We also confirmed the essential role of STAT3 activation blockage in the anti-melanoma action of DPH (Fig. 4). Constitutive STAT3 activation is highly correlated with tumorigenesis, progression and drug resistance in a variety of human tumors, including melanoma (16-18). In contrast, ablating STAT3 activation has been shown to inhibit tumor growth, retard metastasis and overcome resistance to drugs such as vemurafenib (25), highlighting that STAT3-targeted drugs are promising cancer therapeutics. Along this line, DPH acting as an inhibitor of STAT3 activation holds great potential as an anticancer agent. The mechanisms whereby DPH supresses STAT3 activation remain elusive and are currently under investigation in our laboratory.

In the present study, we confirmed that DPH impairs STAT3 activation thus blocking STAT3-mediated induction of MCL-1 (Fig. 4), indicating that DPH targets the STAT3/ MCL-1-mediated survival signaling pathway triggering melanoma cell apoptosis. Several lines of evidence have underscored the central role of the STAT3/MCL-1 signaling axis in the progression and survival of melanoma cells. In melanoma tumor samples, the levels of p-Y705-STAT3 and MCL-1 were shown to be increased together in association with melanoma progression (12). Furthermore, blocking STAT3 activation led to apoptosis in melanoma cells accompanied by downregulation of MCL-1 (16). Moreover, recent studies have validated that oncogenic $\mathrm{BRAF}^{\mathrm{V} 600 \mathrm{E}}$-initiated survival signaling in melanoma cells actually depends on STAT3-mediated MCL-1 upregulation, as blockade of STAT3 activation impairing the induction of MCL-1 by BRAF ${ }^{\mathrm{V} 600 \mathrm{E}}$ and consequently leading to massive apoptosis (15). Thus, drugs such as DPH targeting the STAT3/MCL-1 survival signaling pathway should hold great potential as novel therapeutic agents for the treatment of melanoma.

In conclusion, we herein establish DPH as a selective apoptosis inducer of melanoma cells through targeted suppression 
of the STAT3/MCL-1 survival signaling pathway. The in vivo anti-melanoma activity of DPH was also clearly validated. Our discovery therefore suggests the possible clinical application to repurpose DPH as a novel cancer therapeutic agent in the treatment of melanoma.

\section{Acknowledgements}

This study was supported by the Ministry of Education, Taiwan, R.O.C. under the ATU plan. The authors are indebted to Professor Chi-Chen Lin (National Chung Hsing University) for his generous assistance in the in vivo B16-F10 melanoma growth study.

\section{References}

1. Friedman RJ and Heilman ER: The pathology of malignant melanoma. Dermatol Clin 20: 659-676, 2002.

2. Lens MB and Dawes M: Global perspectives of contemporary epidemiological trends of cutaneous malignant melanoma. Br J Dermatol 150: 179-185, 2004.

3. Houghton AN and Polsky D: Focus on melanoma. Cancer Cell 2: 275-278, 2002

4. Diepgen TL and Mahler V: The epidemiology of skin cancer. $\mathrm{Br}$ J Dermatol 146 (Suppl 61): 1-6, 2002.

5. Linos E, Swetter SM, Cockburn MG, Colditz GA and Clarke CA Increasing burden of melanoma in the United States. J Invest Dermatol 129: 1666-1674, 2009.

6. Antihistamine Drugs: Drug information 85. American Society of Hospital Pharmacists, Bethesda, MD, 1985.

7. Dupuis LL and Nathan PC: Optimizing emetic control in children receiving antineoplastic therapy: Beyond the guidelines. Paediatr Drugs 12: 51-61, 2010.

8. Kuo CC, Huang RC and Lou BS: Inhibition of $\mathrm{Na}^{+}$current by diphenhydramine and other diphenyl compounds: Molecular determinants of selective binding to the inactivated channels. Mol Pharmacol 57: 135-143, 2000.

9. Green SM, Rothrock SG and Gorchynski J: Validation of diphenhydramine as a dermal local anesthetic. Ann Emerg Med 23: 1284-1289, 1994.

10. Jangi SM, Díaz-Pérez JL, Ochoa-Lizarralde B, Martín-Ruiz I, Asumendi A, Pérez-Yarza G, Gardeazabal J, Díaz-Ramón JL and Boyano MD: H1 histamine receptor antagonists induce genotoxic and caspase-2-dependent apoptosis in human melanoma cells. Carcinogenesis 27: 1787-1796, 2006

11. Hsieh HY, Shieh JJ, Chen CJ, Pan MY, Yang SY, Lin SC, Chang JS, Lee AYL and Chang CC: Prodigiosin down-regulates SKP2 to induce $\mathrm{p} 27^{\mathrm{KIP} 1}$ stabilization and antiproliferation in human lung adenocarcinoma cells. Br J Pharmacol 166: 2095-2108, 2012.
12. Zhuang L, Lee CS, Scolyer RA, McCarthy SW, Zhang XD, Thompson JF and Hersey P: Mcl-1, Bcl-XL and Stat 3 expression are associated with progression of melanoma whereas Bcl-2, AP-2 and MITF levels decrease during progression of melanoma. Mod Pathol 20: 416-426, 2007.

13. Perciavalle RM and Opferman JT: Delving deeper: MCL-1's contributions to normal and cancer biology. Trends Cell Biol 23: 22-29, 2013.

14. Belmar J and Fesik SW: Small molecule Mcl-1 inhibitors for the treatment of cancer. Pharmacol Ther 145: 76-84, 2015.

15. Becker TM, Boyd SC, Mijatov B, Gowrishankar K, Snoyman S, Pupo GM, Scolyer RA, Mann GJ, Kefford RF, Zhang XD, et al: Mutant B-RAF-Mcl-1 survival signaling depends on the STAT3 transcription factor. Oncogene 33: 1158-1166, 2014.

16. Niu G, Bowman T, Huang M, Shivers S, Reintgen D, Daud A Chang A, Kraker A, Jove R and Yu H: Roles of activated Src and Stat3 signaling in melanoma tumor cell growth. Oncogene 21: 7001-7010, 2002.

17. Siveen KS, Sikka S, Surana R, Dai X, Zhang J, Kumar AP, Tan BKH, Sethi G and Bishayee A: Targeting the STAT3 signaling pathway in cancer: Role of synthetic and natural inhibitors. Biochim Biophys Acta 1845: 136-154, 2014.

18. Zhao C, Li H, Lin HJ, Yang S, Lin J and Liang G: Feedback activation of STAT3 as a cancer drug-resistance mechanism. Trends Pharmacol Sci 37: 47-61, 2016.

19. Mukherjee N, Lu Y, Almeida A, Lambert K, Shiau CW, Su JC, Luo Y, Fujita M, Robinson WA, Robinson SE, et al: Use of a MCL-1 inhibitor alone to de-bulk melanoma and in combination to kill melanoma initiating cells. Oncotarget: Apr 12, 2016 (Epub ahead of print).

20. Jangi SM, Asumendi A, Arlucea J, Nieto N, Perez-Yarza G, Morales MC, de la Fuente-Pinedo M and Boyano MD: Apoptosis of human T-cell acute lymphoblastic leukemia cells by diphenhydramine, an H1 histamine receptor antagonist. Oncol Res 14: 363-372, 2004.

21. Jangi SM, Ruiz-Larrea MB, Nicolau-Galmés F, Andollo Y, Arroyo-Berdugo N, Ortega-Martínez I, Díaz-Pérez JL, and Boyano MD: Terfenadine-induced apoptosis in human melanoma cells is mediated through $\mathrm{Ca}^{+2}$ homeostasis modulation and tyrosine kinase activity, independently of $\mathrm{H} 1$ histamine receptors. Carcinogenesis 29: 500-509, 2008.

22. García-Quiroz J and Camacho J: Astemizole: An old antihistamine as a new promising anti-cancer drug. Anticancer Agents Med Chem 11: 307-314, 2011.

23. Roger S, Gillet L, Le Guennec JY and Besson P: Voltage-gated sodium channels and cancer: Is excitability their primary role? Front Pharmacol 6: 152, 2015.

24. Fofaria NM, Frederick DT, Sullivan RJ, Flaherty KT and Srivastava SK: Overexpression of Mcl-1 confers resistance to $\mathrm{BRAF}^{\mathrm{V} 600 \mathrm{E}}$ inhibitors alone and in combination with MEK1/2 inhibitors in melanoma. Oncotarget 6: 40535-40556, 2015.

25. Liu F, Cao J, Wu J, Sullivan K, Shen J, Ryu B, Xu Z, Wei W and Cui R: Stat3-targeted therapies overcome the acquired resistance to vemurafenib in melanomas. J Invest Dermatol 133: 2041-2049, 2013. 